\title{
Phase Preserving Amplitude Saturation through Tone Synthesis Assisted Saturated Four-Wave Mixing
}

\author{
K. R. H. Bottrill, N. Taengnoi, Y. Hong, D. J. Richardson, P. Petropoulos
}

\begin{abstract}
When processing a data carrying signal with amplitude saturation in a fibre-optic parametric amplifier, the signal can be subject to a deleterious conversion of its amplitude noise into phase noise. To overcome this, we propose and demonstrate a scheme in which the products of four-wave mixing are instead synthesised using spectral shaping of multicast replicas of the signal, rather than naturally through four-wave mixing itself. This allows us to emulate the advancement of four-wave mixing without incurring unwanted amplitude noise to phase noise conversion. A final stage of four-wave mixing is used to bring about amplitude squeezing, with little impact on the phase noise of the signal. Using this technique, we are able to demonstrate phase preserving amplitude squeezing of a $10 \mathrm{GBd}$ QPSK signal using as a medium, a highly nonlinear fibre in which the traditional, single pump approach fails.
\end{abstract}

\section{INTRODUCTION}

Amplitude regeneration [1]-[3] is an essential member of our developing all-optical signal processing toolkit and, along with phase regeneration [4], [5], phase conjugation [6], [7], harmonic generation [8] and wavelength conversion [9], [10], is an important part of any future all-optical network [11]. There is interest in performing such operations purely in the optical domain owing to the ultra-wide bandwidth, low latency and high energy efficiency that all-optical schemes could offer over optical-electronic-optical (OEO) approaches [12], [13]. Although these operations can be achieved optically through a variety of phenomena, four-wave mixing (FWM) is notable in that it can be used to implement them all.

Many FWM based all-optical processing schemes, despite being effective at carrying out their desired function, often exhibit some deleterious amplitude to phase noise conversion (ANPNC), unless particular mitigating steps are taken. FWM based amplitude saturation is no different. By studying the Bessel formulation of FWM [14], [15], we showed in [16], [17] that the extent of this ANPNC is determined by the pump to signal power ratio used to achieve signal amplitude squeezing and that by selecting as high a pump to signal power ratio as possible (assuming a noiseless pump), ANPNC can be suppressed. Whilst effective, this approach is subject to all of the challenges associated with launching a powerful continuous wave $(\mathrm{CW})$ pump into a highly nonlinear fibre

The data for this work is accessible through the University of Southampton Institutional Research Repository (DOI:10.5258/SOTON/xxxxxx).

This work was supported by the Engineering and Physical Sciences Research (EPSRC) project PHOS under Grant EP/S002871/1.

The authors are affiliated with the Optoelectronics Research Centre, University of Southampton, SO17 1BJ, UK.
(HNLF), namely the generation of low noise pumps with the necessary high power as well as the need to mitigate adverse nonlinear effects, such as stimulated Brillouin scattering (SBS). Furthermore, once processing of the optical signal is complete, there is a considerable amount of optical power which will be discarded as the regenerated signal is extracted through filtering, limiting the efficiency of such processing approaches.

To overcome the challenges associated with using high pump to signal power ratios, we later demonstrated a twostage scheme to achieve amplitude saturation [3]. In that work, we used an initial stage of FWM to produce a conjugate of the signal which was loaded with phase noise due to selfphase modulation (SPM). A second conjugating stage of FWM was then finally used to achieve amplitude saturation whilst simultaneously undoing the SPM induced distortion of the first stage in a process similar to optical phase conjugation. As the two stages of SPM counteract each other, very much lower pump to signal power ratios can be used without a reduction in performance, permitting the use of much lower pump powers and hence reducing the complexity of the system. This system was less challenging to implement but of course did require two FWM stages along with the additional optical componentry.
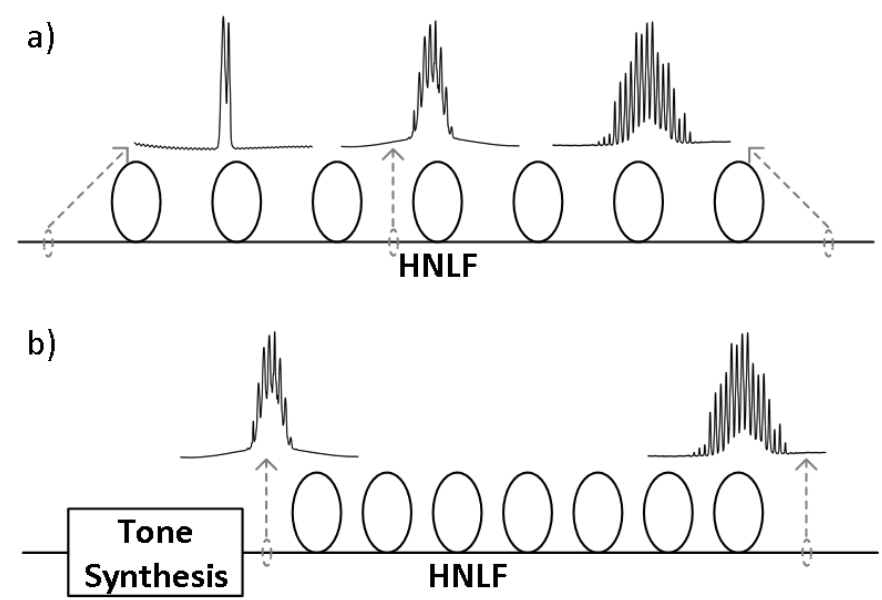

Fig. 1. Illustration of a) typical approach to FWM based amplitude saturation b) the proposed, tone-synthesis based approach.

In this work (an extension of [18]), we present a new, 'pumpless' approach to amplitude squeezing, which offers low power operation with low ANPNC, all the while circumventing 
any impediments due to SBS. Amplitude squeezing through FWM can be considered to consist of two stages - an initial, harmonic generating stage and a final amplitude saturating stage, both of which take place consecutively in the same fibre. In this work, we substitute the FWM-based harmonic generating stage with a Mach-Zehnder modulator (MZM) and a programmable optical filter $(\mathrm{PF})$ which are used to synthesise the required harmonic state before saturation. This state of synthesised harmonics is then launched into an HNLF so that the actual amplitude saturating stage can take place as normal. The use of a MZM circumvents the ANPNC that the signals would experience using the typical FWM based approach and as the 'pump' is essentially derived from one of the sidebands produced by the MZM, no additional laser sources are required. Furthermore, SBS is largely overcome, not only because the technique requires relatively low powers to be launched into the FWM stage, but also because the 'pump' which normally consists of a modulated, data-carrying signal, will typically have an effective bandwidth many times that of the Brillouin gain bandwidth of $20-50 \mathrm{MHz}$ [19].

We firstly demonstrate that it is possible to synthesise the harmonics as described and that when they are launched into the HNLF, FWM does indeed progress as expected. We then apply the scheme to the regeneration of a $10 \mathrm{GBd}$ QPSK signal, providing constellation plots, noise statistics and BERs, which we compare to the results obtained from a conventional, single-pump saturated FWM system. Our proposed scheme allows us to demonstrate phase preserving amplitude regeneration where the traditional, single-pump scheme fails.

\section{BACKGROUND}

A generalised schematic for achieving FWM-based amplitude saturation is provided in Fig. 1-a) and coarsely, it can be said that amplitude saturation is achieved by multiplexing a pump with the signal to be regenerated and increasing the power of either the signal, pump or both until saturation occurs. A much better understanding of the process can be gained through mathematical analysis. In a dispersionless fibre, the longitudinal evolution of a pump, a signal and the generated harmonics due to degenerate FWM can readily be described by a Bessel formulation [14]-[17]:

$$
\begin{gathered}
\sqrt{P_{m}^{\prime}} e^{i\left(\omega_{m}^{\prime} t+\phi_{m}^{\prime}\right)=} e^{i \gamma L P_{0}} e^{i \gamma L P_{1}} e^{i\left[m\left(\omega_{1} t+\phi_{1}\right)-(m-1)\left(\omega_{0} t+\phi_{0}\right)\right]} \\
\left\{i^{m} \sqrt{P_{0}} J_{m}\left(2 \sqrt{P_{0} P_{1}} \gamma z\right)\right. \\
\left.+i^{m-1} \sqrt{P_{1}} J_{m-1}\left(2 \sqrt{P_{0} P_{1}} \gamma z\right)\right\}
\end{gathered}
$$

where the subscript $m$ corresponds to the order of the harmonic in question ( $m=1$ for the signal, $m=0$ for the pump and negative values of $m$ for conjugated tones), $P_{m}$, $\omega_{m}, \phi_{m}$ is the power, radial frequency and phase of the $m$ th harmonic, $\gamma$ is the nonlinear coefficient of the fibre, $z$ is the distance along the length of the fibre and $J_{n}(x)$ is a Bessel function of the first kind. Primed terms correspond to values at point $z$ along a fibre, whilst unprimed terms correspond to their initial values. In short, this equation shows that the evolution of every tone in the system can be described by the sum of two Bessel functions of the first kind, with argument $2 \sqrt{P_{0} P_{1}} \gamma z$. Cross-phase modulation (XPM) and SPM on the signal can be seen in the terms $e^{i \gamma z P_{0}}$ and $e^{i \gamma z P_{1}}$, respectively and in [16], [17], we identified the relative change in power between the two orthogonal Bessel functions to cause a further phase modulation, which we named Bessel order mixing (BOM). SPM and BOM are the predominant causes of ANPNC [16], [17] and we previously showed that they both can be suppressed by adopting a high pump to signal power ratio. In the limit of high pump to signal power ratio (where $\left.P_{0}>>P_{1}\right)$, Eq. 1 becomes:

$$
\begin{aligned}
\sqrt{P_{m}^{\prime}} e^{i\left(\omega_{m}^{\prime} t+\phi_{m}^{\prime}\right)}=e^{i \gamma L P_{0}} & e^{i\left[m\left(\omega_{1} t+\phi_{1}\right)-(m-1)\left(\omega_{0} t+\phi_{0}\right)\right]} \\
& \times\left\{i^{m} \sqrt{P_{0}} J_{m}\left(2 \sqrt{P_{0} P_{1}} \gamma L\right)\right\}
\end{aligned}
$$

with the corresponding power evolution (obtained by taking the modulus squared of Eq. 2) described by:

$$
P_{m}^{\prime}=P_{0} J_{m}^{2}\left(2 \sqrt{P_{0} P_{1}} \gamma z\right)
$$

and ANPNC vanishes [16].
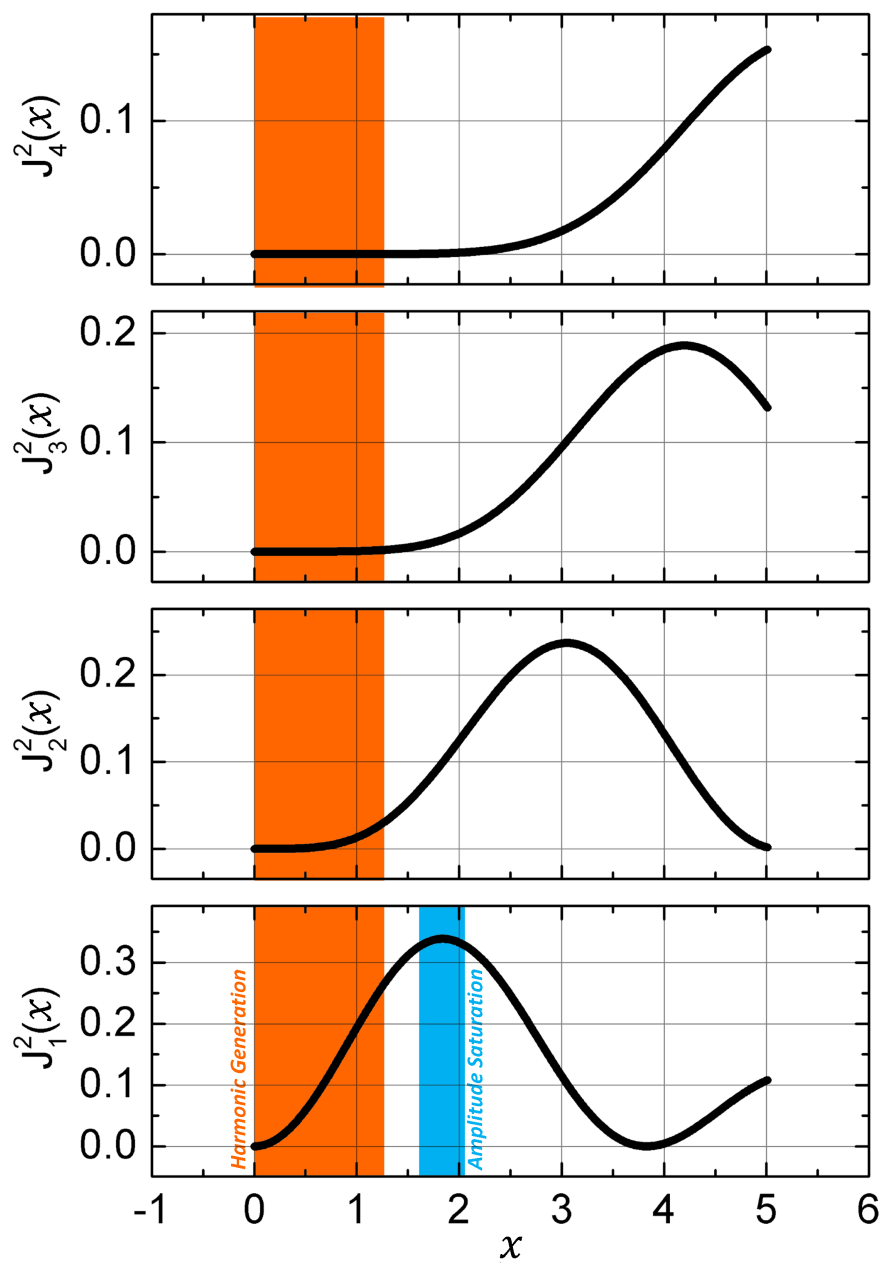

Fig. 2. Bessel functions of the first kind for orders 1 to 4 . Harmonic generation stage is highlighted in orange, amplitude saturation of the signal is highlighted in blue.

Fig. 2 provides plots of squared Bessel functions of the first kind with order between 1 and $4\left(J_{1}^{2}(x), J_{2}^{2}(x), J_{3}^{2}(x)\right.$ 
and $\left.J_{4}^{2}(x)\right)$. Note, that in the system described by Eq. 3, $x$ can be considered equal to $2 \sqrt{P_{0} P_{1}} \gamma z$. Highlighted in blue for $J_{1}(x)$ is the region in which the first maximum lies and about which amplitude squeezing can take place. The peak in $J_{1}(x)$ occurs for a value of $x=1.84$ [16] and from Eq. 3, implies that the necessary condition for amplitude saturation of the signal is $2 \sqrt{P_{0} P_{1}} \gamma z=1.84$. In other words, when the tones exit the fibre at $z=L$ (the total length of the optical fibre), $2 \sqrt{P_{0} P_{1}} \gamma L=1.84$ must be true in order for the signal to leave the fibre with its amplitude saturated. In its simplest implementation wherein only the first peak of the Bessel function is used to squeeze the amplitude of a signal, the system is intrinsically applicable only to single power level signals, such as M-PSK formats although use of higher order peaks may facilitate its application to multi-level formats. Polarisation multiplexed signals can, however, be supported using a polarisation diversity scheme [20]. Techniques for all-optical regeneration not based on FWM include nonlinear optical loop mirror based approaches for both single [21] and multi-level signals [1], [22], single-level saturation in semiconductor optical amplifiers [23] as well as multi-level regeneration in a Mamyshev regenerator [24].

Although effective, raising the pump to signal power ratio to suppress ANPNC is not without its practical challenges, which we shall prove in this section. Indeed, we shall show that, for a typical Germanium doped HNLF, with no additional steps taken to raise its Brillouin threshold, it is not possible to achieve an acceptably low ANPNC, even when using the maximum pump power allowed for by SBS.

We assume, a posteriori, that the system will saturate when $P_{1}>P_{0}$ and hence from [16], we know that saturation will occur when $2 \sqrt{P_{0} P_{1}} \gamma L=1.26$ (in contrast with the case above for high pump to signal power ratio where $\left.2 \sqrt{P_{0} P_{1}} \gamma L=1.84\right)$. Defining the pump to signal power ratio at saturation to be $R=P_{0} / P_{1}$ and setting the pump power to be equal to the Brillouin threshold (the maximum usable power), $P_{0}=P_{t h}$, the saturation condition with maximal pump power becomes $2 P_{t h} \sqrt{1 / R} \gamma L=1.26$ which can be rearranged for $R$ as:

$$
R=\left(\frac{2 P_{t h} \gamma L}{1.26}\right)^{2}
$$

Meanwhile, from [25] (and assuming no polarisation maintenance in the fibre, which results in the highest Brillouin threshold - the best case scenario) we can formulate the Brillouin threshold, $P_{t h}$, of the fibre as:

$$
P_{t h}=\frac{42 A_{e f f}}{g_{B} L}
$$

Substituting Eq. 5 into Eq. 4 we obtain:

$$
R=\left(\frac{84 A_{e f f} \gamma}{1.26 g_{B}}\right)^{2}
$$

Finally, substituting in the definition of the nonlinear coefficient, $\gamma=\frac{n_{2} \omega}{c A_{e f f}}$ [26] allows us to make some important simplifications:

$$
R=\left(\frac{84 n_{2} \omega}{1.26 g_{B} c}\right)^{2}
$$

From Eq. 7 we can already draw some notable conclusions, namely, that $R$, which must be maximised to minimise ANPNC, depends neither upon the length, $L$, nor upon the effective area, $A_{e f f}$, of the medium; it is only the ratio of nonlinear index to Brillouin gain coefficient, $n_{2}: g_{b}$ which determines ANPNC.

Substituting in the following parameters typical of a Germanium doped HNLF: $n_{2} \approx 2.96 \times 10^{-20} \mathrm{~m}^{2} \mathrm{~W}^{-1}$ [27] and $g_{B} \approx 4 \times 10^{-11} \mathrm{~mW}^{-1}$ [25] and considering light of wavelength $1550 \mathrm{~nm}$, we find that $R=0.04$ or equivalently $-14 \mathrm{~dB}$. In other words, even with maximum pump power permitted by SBS, saturation is only achieved when the signal is $14 \mathrm{~dB}$ above the pump. In [16] we experimentally demonstrated that, for acceptably low ANPNC, the pump to signal power ratio should be considerably greater than $0 \mathrm{~dB}$ (indeed, ideally it should be above $10 \mathrm{~dB}$ ). Therefore, the conclusion is that, without the adoption of SBS suppression schemes, it is impossible to achieve acceptable performance using the conventional scheme in a typical Germanium doped silica HNLF. This provides our motivation to investigate alternative schemes to reduce ANPNC and/or overcome SBS.

\section{Proposed Scheme}

Returning to Fig. 2, the harmonic generation stage has been highlighted in orange, and it can be seen to correspond to an increasing, mostly linear region of $J_{1}(x)$ during which time the higher order Bessel modes slowly increase in power. It is clear that although no amplitude squeezing takes place in this region, amplitude saturation cannot take place until there is sufficient power in the higher harmonics - the generation of which implies a concomitant accrual of ANPNC due to SPM and BOM as the pump, signal and harmonics propagate along the processing fibre. It is this region during which no amplitude squeezing takes place and during which the majority of the ANPNC occurs that we wish to bypass by synthesising the harmonics described by Eq. 3 using some other equivalent means.

Considering the Kerr effect to be effectively instantaneous, the system is agnostic as to how a field at a particular time and location in the fibre, $A(z, t)$ came to be (contrast this with, for instance, carrier depletion induced nonlinearity in semiconductor optical amplifiers which occurs over much longer timescales). This indicates that we can synthesise it by any means we choose, a principle illustrated by the schematic in Fig. 1-b). In this work, we choose to synthesise the harmonics by producing a series of replicas of the original signal in the frequency domain (akin to a frequency comb, but with data carrying signals rather than monochromatic tones) and conditioning the resultant tones using a PF so that they follow the relative powers described by Eq. 3. These two processing elements are essentially agnostic as to the nature of the signals being processed - the MZM simply modulates the incoming light to produce a frequency comb whilst the PF applies a static, frequency dependent attenuation to the signal. 


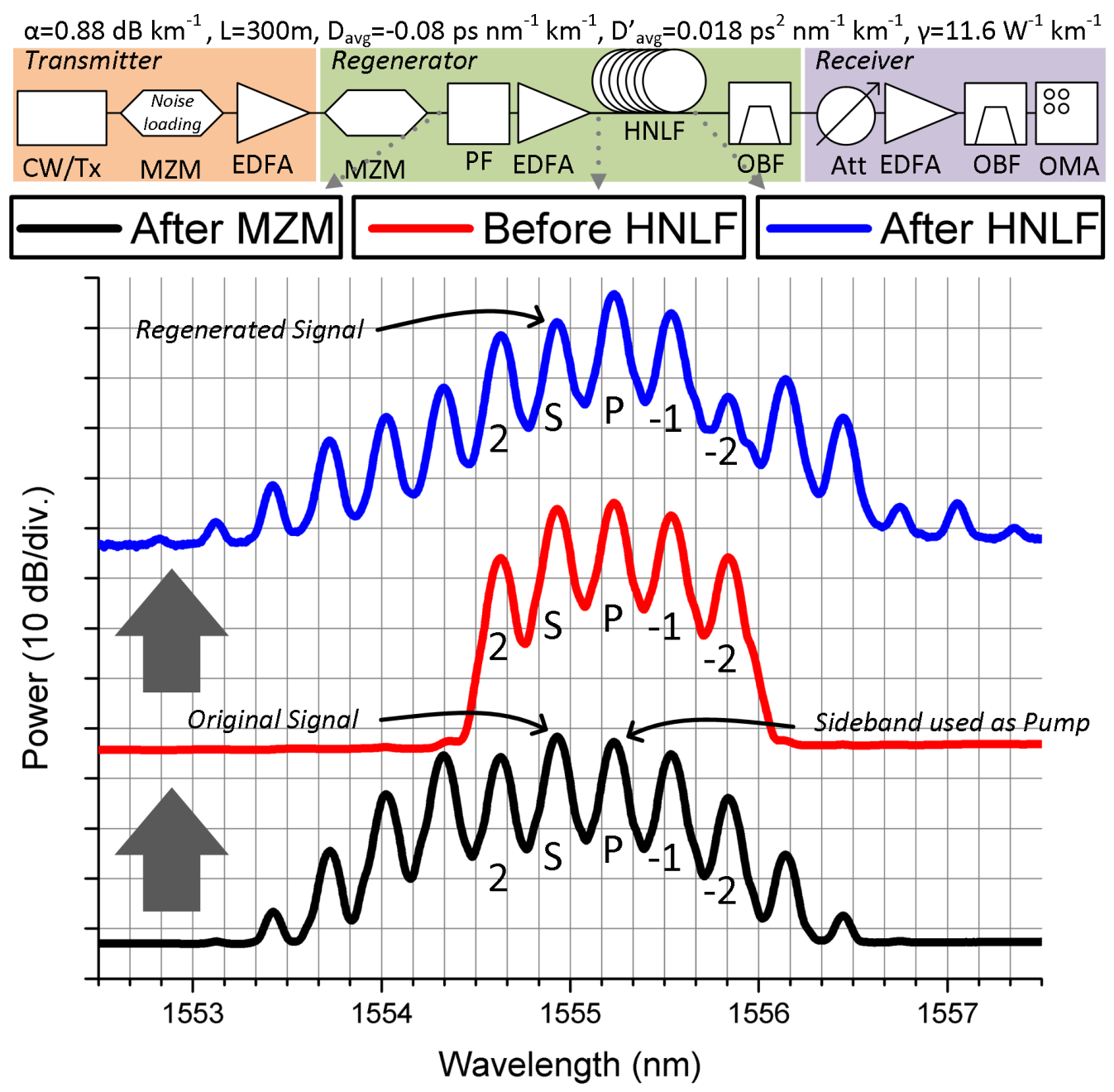

Fig. 3. Top: Experimental set-up Bottom: Spectra at the identified stages of the set-up.

In other words, whilst we can replace the harmonic generating stage using these two elements, we cannot replace the final, amplitude saturating stage - for that, we still need to rely on FWM. Note, that the proposed scheme relies on multicasting the signal in the frequency domain by some method - this multicasting need not be achieved using a MZM and indeed other methods are applicable too. For instance, an overdriven phase modulator may be used to produce the multicast signals in much the same way as the MZM; the choice will come down to practical considerations, for instance, phase modulators require no bias control.

One important and notable difference between this synthesised system and the conventional system is the nature of the 'pump'. In the traditional system, the pump is a separate CW tone which is multiplexed with the signal to be processed. In contrast, the 'pump' in our proposed scheme is formed from one of the tones produced by the MZM, hence our description of the scheme as 'pumpless' - no additional optical sources are needed to process the signal beside the signal itself. The benefit in reducing system complexity is twofold - not only are the high power, narrow linewidth pumps normally needed for low ANPNC obsolete but also, because the pump is essentially a wavelength converted replica of the original, modulated signal, the spectral breadth of the pump acts to increase its effective SBS threshold. Note that, as the tones produced by the MZM are essentially identical, we can choose any we like to constitute the pump. Naturally, we choose a tone adjacent to the location of the original signal, so that the processed output signal lies at the exact same wavelength as the original input to the system. An important point to consider is the phase relationship between the tones. Degenerate FWM is known to result in the following phase relationship between consecutive tones: $\phi_{m+1}=2 \phi_{m}-\phi_{m-1}$. By assuming all tones produced by the MZM have the same phase, this condition is automatically replicated. Furthermore, we assume the selection of a low dispersion HNLF as the processing medium. In practice, although the dispersion is unlikely to be truly zero, any deviation should manifest as a small perturbation, mainly resulting in a reduction of phase matching and hence FWM efficiency. In [16] we showed that the dispersionless model above matched very well with experimental measurements, only suffering errors when predicting the highest harmonic orders measured $(n>4)$. If the improvement of FWM efficiency is desired, any phase error between the tones due 
a)

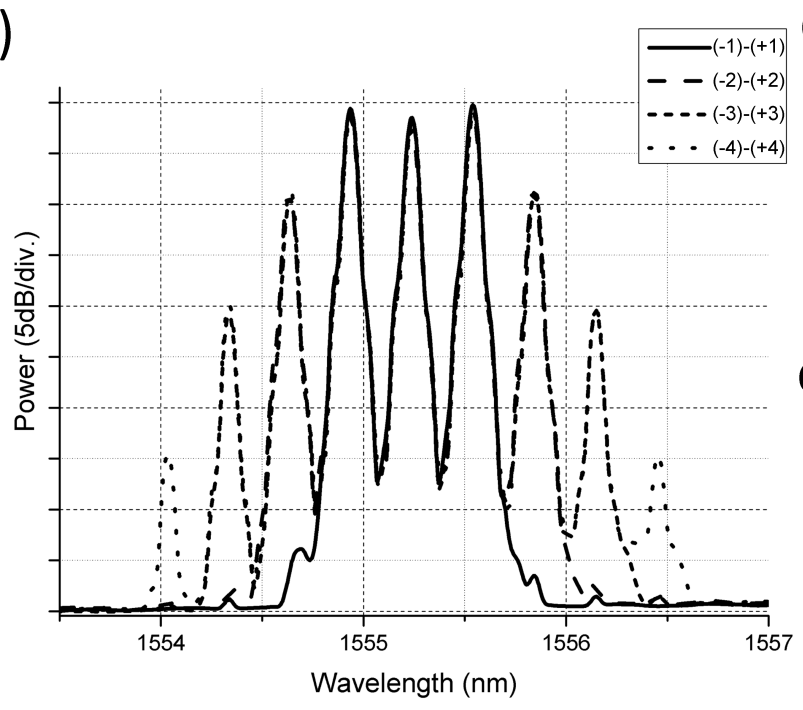

b)

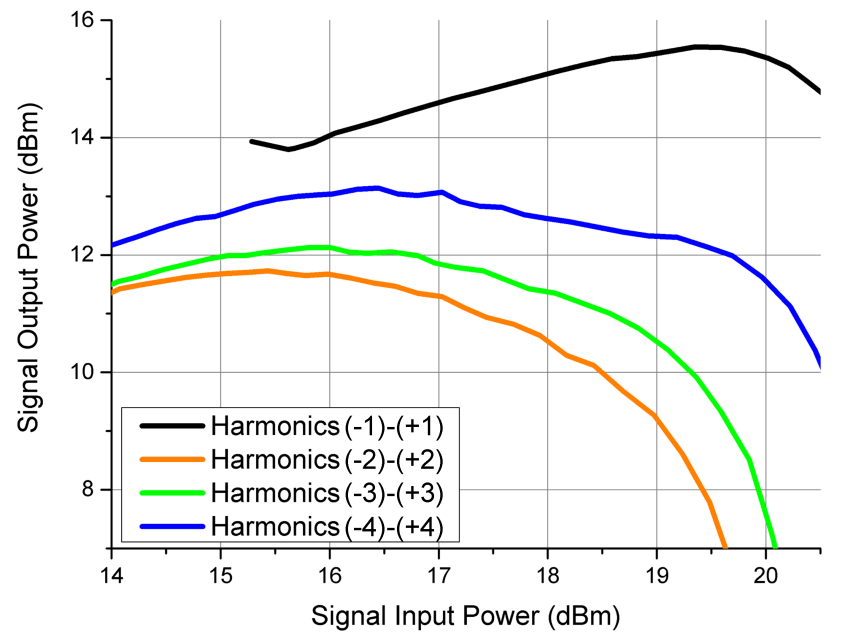

c)

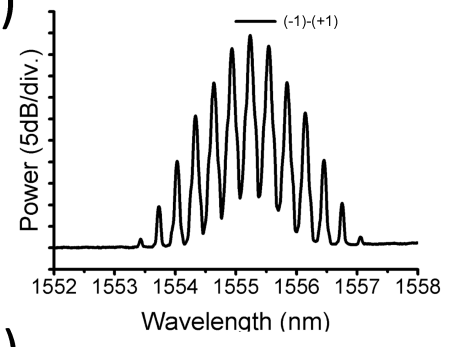

d)

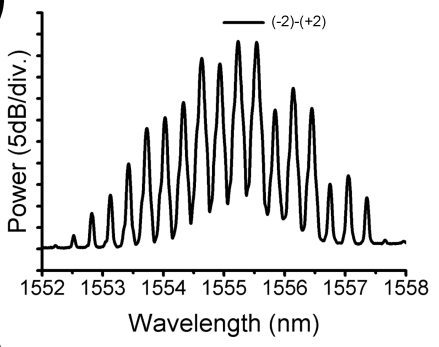

e)

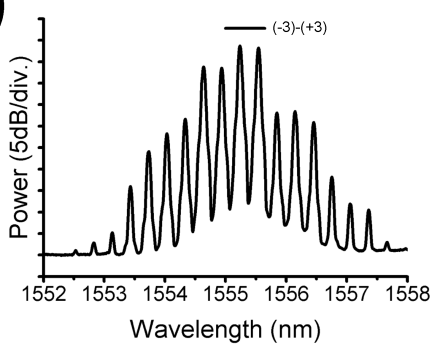

f)

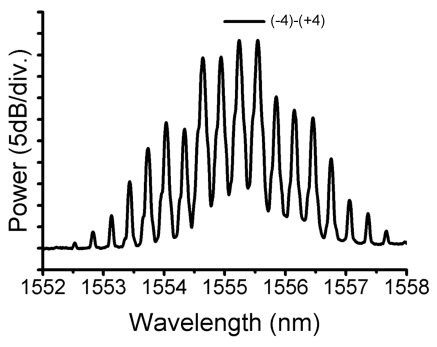

Fig. 4. a) Spectra of synthesised launch waves for harmonic orders from 1 to 4 ; b) Signal output power as it varies with signal input power for harmonic orders from 1 to 4 ; c) Output spectra for the $(-1)-(+1)$ case, d) Output spectra for the $(-2)-(+2)$ case, e) Output spectra for the $(-3)-(+3)$ case, f) Output spectra for the $(-4)-(+4)$ case.

to the HNLF or any other part of the system could be compensated by using the phase shifting functionality of a so capable PF, but for the sake of simplicity, we do not adopt such a technique here.

All in all, the system allows us to achieve low ANPNC amplitude squeezing in a relatively low power system with no need for additional light sources and an intrinsically low susceptibility to SBS.

\section{EXPERIMENTAL SET-UP}

The experimental set-up used in this study is provided in Fig. 3. The diagram has been divided into 3 sections, the transmitter (orange), the regenerator (green) and the receiver (purple). The transmitter generates either a $\mathrm{CW}$ test signal, or a $10 \mathrm{GBd}$ single polarisation QPSK signal which first passes through a MZM so that a controllable amount of amplitude noise can be applied to it, for characterisation. The signal is then launched into an EDFA to compensate for the loss in the noise loading stage.

Within the regenerator, the signal first passes through the MZM (with an insertion loss of $4 \mathrm{~dB}$ ) which is used to create a series of tones in the frequency domain with a spacing of 37.5GHz (see black trace in Fig. 3). This tone spacing was used as it is equal to an integer multiple of the granularity of the PF and hence allows for the most accurate production of the desired optical response. The PF (a wavelength selective switch with an insertion loss of $5 \mathrm{~dB}$ ) is used to filter the tones so that their relative powers agree with Eq. 3, as desired. After passing through the PF, the signals are first amplified to a moderate power level (around $21 \mathrm{dBm}$, depending upon the scenario being tested) before passing through the HNLF to undergo FWM (the red spectrum in 
Fig. 3 shows an example spectrum which might be carved from the frequency comb). The fibre is a $300 \mathrm{~m}$, dispersion flattened, strained HNLF, with the following parameters (measured at 1550nm): $\alpha=0.88 \mathrm{dBkm}^{-1}, D=-0.08 \mathrm{psnm}^{-1} \mathrm{~km}^{-1}$, $D^{\prime}=0.018 \mathrm{ps}^{2} \mathrm{~nm}^{-1} \mathrm{~km}^{-1}, \lambda_{0}=1554.44 \mathrm{~nm}$ and $\gamma=$ $11.6 \mathrm{~W}^{-1} \mathrm{~km}^{-1}$ (see blue spectrum in Fig. 3 for an example of the output of the HNLF). After mixing, the processed signal is selected using an optical bandpass filter, after which it enters the receiver.

The receiver consists of a variable attenuator followed by an EDFA, an optical bandpass filter to reject ASE and finally a coherent receiver. The combination of variable attenuator and EDFA allows for the control of the received OSNR so that BERs can be measured.

\section{RESULTS}

The system was first characterised using a CW laser as its input signal so that re-establishment of the evolution described by Eq. 3 could be verified. Although a wave-based formulation of FWM involves the evolution of an infinite series of harmonics, we restrict ourselves to approximations involving a finite number of harmonics centred around the pump. This not only makes the technique more accessible, both for ourselves and for future implementers, but also helps to maximise OSNR; the accurate replication of the relative powers described by Eq. 3 often requires attenuation of many of the tones, with a resultant decrease in OSNR. We justify this truncation by positing that, because the power of the harmonics decreases with increasing absolute harmonic order, the harmonics become increasingly less important as their order increases. Of note, Matsumoto has shown that good approximation to saturation in FWM can be obtained using a semi-analytical approach considering only the pump, signal, the signal's idler and the second harmonic [28].

There are two main dependent variables that are of interest to us: the length of the truncated harmonic series and the value of $x$ emulated, both adjusted using the PF. Note that, to achieve saturation, we must choose values of $x$ that are $<1.84$; we should not be under any illusion, that by tuning the relative powers of the tones to follow $J_{m}^{2}(1.84)$, we would be able to squeeze the signal without it ever undergoing FWM - the action of the MZM is agnostic to the instantaneous phase of the input signal and therefore incapable of achieving this.

We firstly study what effect the length of the truncated series has upon the behaviour of the system. To do so, we use the MZM and PF to produce a series of harmonics whose relative power can be described by $J_{m}^{2}(x)$, where $x=1.5$. The value of $x=1.5$ is chosen as it is near but just below the power required for saturation (which lies at $x=1.84$ ), but still relieves the fibre of producing a substantial amount of FWM. We will consider consecutive series of harmonics, from harmonic $p$ to harmonic $q$ and represent such a scenario as the case ' $(p)-(q)$ ', such that ' $(-2)-(+2)$ ' represents the case where the following set of harmonics are included: -2 , $-1,0,1,2$. Fig. 4 provides results for the following cases: (1)-(+1), (-2)-(+2), (-3)-(+3), and (-4)-(+4). In Fig. 4-a, spectra are overlaid for all four scenarios to show that, aside from the

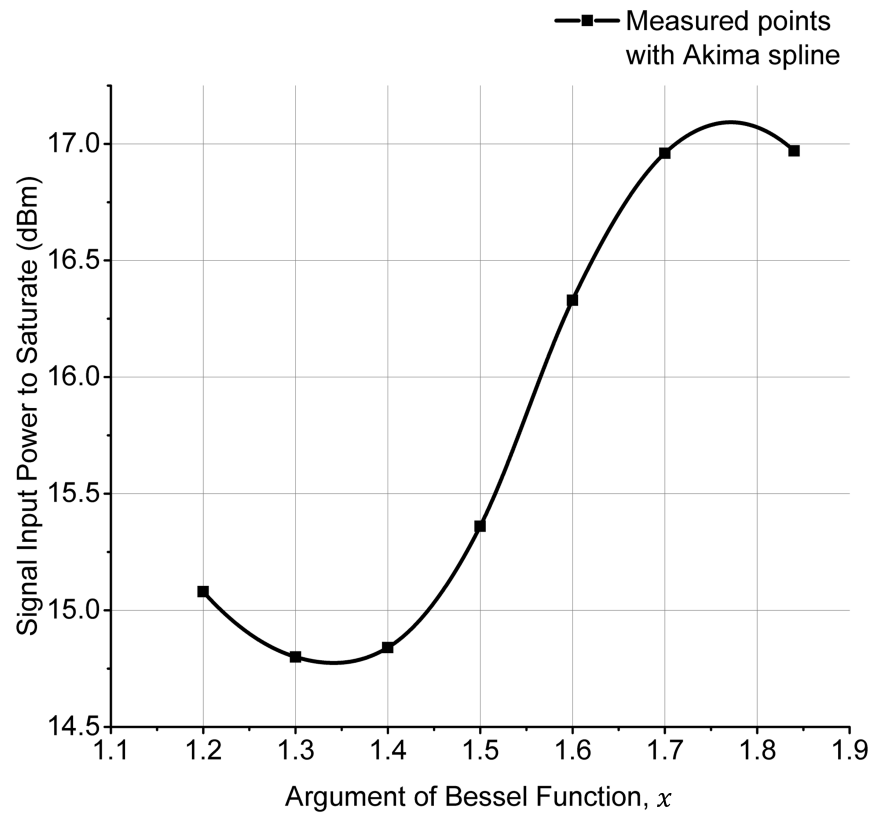

Fig. 5. Input signal power at saturation as it varies with the argument, $x$, of the Bessel functions used to determine harmonic powers in the synthesised input wave.

omitted tones, the input spectrum is otherwise the same for each scenario. In order to find the location of the saturation region, the total power launched into the HNLF is varied using an EDFA. Fig. 4-b shows the signal output power versus the signal input power for each of the four considered scenarios. Scenarios $(-2)-(+2),(-3)-(+3)$, and $(-4)-(+4)$ all show a reasonably similar behaviour, with a peak in power located around $16 \mathrm{dBm}$ signal input power, meanwhile the $(-1)-(+1)$ case is notably different; here the peak is located at a much higher power of $20 \mathrm{dBm}$. This pattern is repeated in the spectra shown in Figs. $4-\mathrm{c}$, -d, -e, and -f, which correspond to scenarios (1)-(+1), (-2)-(+2), (-3)-(+3), and (-4)-(+4), respectively, after mixing in the HNLF with total launch powers of $\approx 23.3 \mathrm{dBm}$. Like before, we see that scenarios $(-2)-(+2),(-3)-(+3)$, and $(-$ $4)-(+4)$ all show similar spectra, with the $(-1)-(+1)$ case being notably different; the pump can be seen to have been amplified and each successive harmonic is of lower power than that of the order preceding it. These results indicate the importance of the 2 nd and conjugated 2 nd harmonic to the mechanism of saturation and show that the three tone scenario, $(-1)-(+1)$, is a very different system from the others considered, suggesting at least that it is not a good approximation to the system described by the Bessel formulation (too many harmonics have been omitted). These results show that the principle mechanism behind saturation is the interaction between the 5 tones in the $(-2)-(+2)$ case and propose that saturation occurs when the signal and conjugate transition from receiving gain from the pump to delivering gain (and therefore being depleted) to the 2nd and conjugated 2nd harmonic.

Next we study the effect of synthesising launch spectra corresponding to Eq. 3 with different values of $x$, the argument of the Bessel function. Here we consider the (-2)-(+2) scenario, as we have shown it to have similar behaviour for the higher 


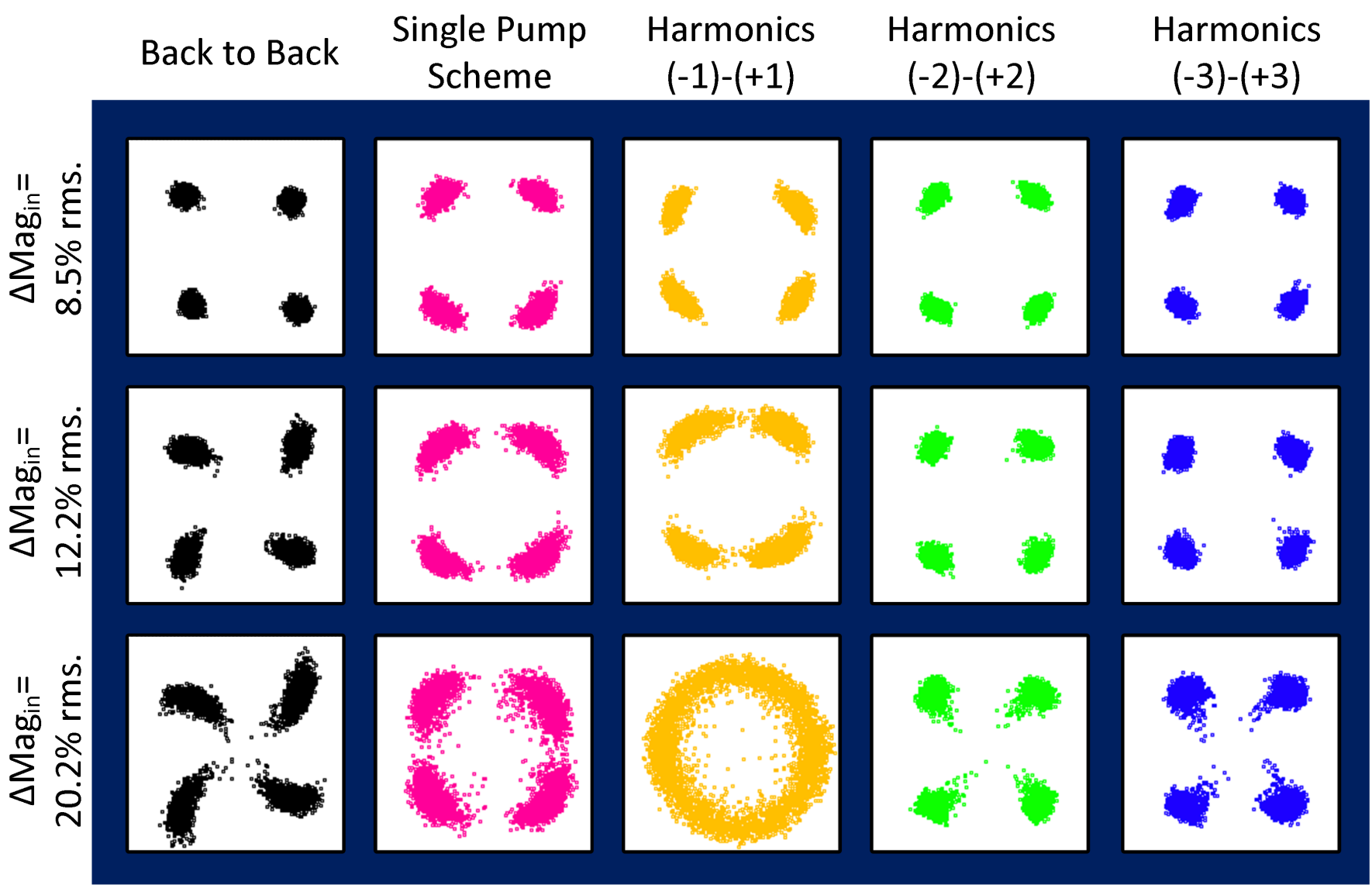

Fig. 6. Constellation plots for 3 input amplitude noise cases: $8.5 \%$ rms., $12.2 \%$ rms. and $20.2 \%$ rms.. Five cases are shown: Column 1$)$ back to back; Column 2) Single Pump scheme; Column 3) $(-1)-(+1)$ case; Column 4) $(-2)-(+2)$ case; Column 5) $(-3)-(+3)$ case.

harmonic cases and spectra which conform with Eq. 3 can be realised with lower OSNR penalty, given the reduced number of tones that need to be generated. The signal output power was measured as it varies with signal input power for different values of $x$. The first peak in power is the scenario desired for squeezing and so these were then identified. Figure 5 provides a plot of the input signal power needed to saturate against the argument of the Bessel function, $x$, experimental points are plotted with squares and have been connected by Akima splines. The lower the signal power used to achieve saturation, the lower the expected ANPNC and so $x$ values located around the minimum of 1.3-1.4 represent good candidate values for this parameter.

With potential operating parameters identified, we move on to demonstrate the performance of the system upon data carrying signals. We replace the $\mathrm{CW}$ wave with a $10 \mathrm{GBd}$ QPSK signal, generated using an IQ modulator driven by two decorrelated PRBS15 drive waveforms. Using a coherent receiver (along with a local oscillator for intradyne detection), regenerative performance was studied using constellation diagrams, noise statistics, and finally, BERs as metrics. For comparison, a simple, single pump scheme (SPS scheme) was implemented by bypassing the MZM and PF used for tone synthesis and instead multiplexing with the signal a CW pump with power held at $21.5 \mathrm{dBm}$, just below the Brillouin threshold of the HNLF. The signal power was increased until saturation was achieved, which occurred for a signal power of $25 \mathrm{dBm}$. According to our work in [16], operating in this regime, with a pump to signal power ratio of $-3.5 \mathrm{~dB}$, should result in unacceptably bad performance. As such, this might seem a poor comparison for our scheme, however, we note that both schemes have been implemented in the exact same fibre with the same extent of Brillouin suppression. Furthermore, the SPS scheme is operated using the maximum pump power possible and likely possesses OSNR advantages relative to the proposed scheme, due to the absence of the losses associated with the MZM and PF.

Figure 6 provides constellation plots for five different systems operating at three different input magnitude noise levels, $\Delta M_{a g_{i n}}=8.5 \%$ rms., $12.2 \% \mathrm{rms}$. and $20.2 \% \mathrm{rms}$. The systems shown are as follows: Column 1 - back to back performance with the signal entering the receiver straight after the noise loading stage (black symbols); Column 2 - SPS scheme (pink symbols); Column 3 - our proposed scheme in the (-1)$(+1)$ configuration (orange symbols), Column 4 - our proposed scheme in the (-2)-(+2) configuration (green symbols), Column 5 - our proposed scheme in the $(-3)-(+3)$ configuration (blue symbols). Comparing the performance of the systems in the first row with $\Delta \mathrm{Mag}_{i n}=8.5 \% \mathrm{rms}$. (corresponding to the case with amplitude noise loading deactivated), we can see that all of the regenerative systems increase phase noise by some margin relative to the back to back case, with the SPS 
scheme and (-1)-(+1) schemes being notably worse than the $(-2)-(+2)$ and (-3)-(+3) schemes. Considering now the second row, with $\Delta \mathrm{Mag}_{\text {in }}=12.2 \%$ rms., we can see that all of the regenerative schemes successfully reduce amplitude noise. However, ANPNC is clearly evident for both the SPS scheme and $(-1)-(+1)$ scheme (the former outperforming the latter), whilst the $(-2)-(+2)$ and $(-3)-(+3)$ schemes show no visible increase in phase noise over the $\Delta M a g_{i n}=8.5 \% \mathrm{rms}$. case. Finally, we consider the $\Delta M a g_{i n}=20.2 \% \mathrm{rms}$. case shown in the third row, which is meant to test the limits of our proposed scheme. The back to back constellation plot shows that this scenario corresponds to a considerable amount of amplitude noise (with concomitant phase noise being due to chirp induced by the single drive, noise loading MZM). Once again, all four regenerative schemes show a reduction in amplitude noise, however, the signal is rendered irrecoverable by the $(-1)-(+1)$ scheme, with the SPS scheme providing the second best performance - the signal is still recoverable but is heavily distorted by phase noise. Once again, the $(-2)-(+2)$ and $(-3)-(+3)$ systems show the best performance, with a notable decrease in amplitude noise and very little increase in phase noise. The output symbol clusters in these cases can be seen to have taken on a somewhat teardrop shape, with a narrower tip directed toward the center of the plot. This narrower tip can be seen to be the remanents of the population of input symbols with the lowest power (seen in the last row of the first column of Figure 6); the power deviation of these symbols relative to the mean symbol power has essentially exceeded the ability of $J_{1}(x)$ function to squeeze. Altogether, these results support the operating philosophy of the scheme - that ANPNC can be reduced by replacing FWM based tone generation by a linear alternative, such as the MZM followed by PF in the present work.

We now move on to consider the change in noise statistics caused by application of these regenerative systems. Fig. 7-a) shows the change in output error vector magnitude $\left(E V M_{\text {out }}\right)$ for each of the four regenerative systems as it varies with $\Delta M a g_{i n}$. Both the SPS system and the (-1)-(+1) system can be seen to result in a large increase in $E V M_{\text {out }}$ with increasing $\Delta M a g_{i n}$, rendering these schemes largely unsuitable for regeneration without some accompanying phase regenerative system to undo the ANPNC caused. The $(-2)-(+2)$ and ($3)-(+3)$ systems on the other hand, result in improvements in $E V M_{\text {out }}$ for all but the lowest $\Delta M a g_{\text {in }}$ case, with the improvement increasing with increasing $\Delta M a g_{i n}$, although the (-2)-(+2) system slightly outperforms the $(-3)-(+3)$ system. Fig. 7-b) shows the output magnitude noise $\Delta M a g_{\text {out }}$ as it varies with $\Delta M a g_{i n}$ and hence shows the regenerator's ability to squeeze the amplitude of the signal. As was indicated by the constellation plots, all schemes are indeed effective at reducing the amplitude noise of the signal, with all schemes offering a greater reduction in $\Delta M a g_{\text {out }}$ with increasing $\Delta M a g_{i n}$. Interestingly, the (-2)-(+2) and (-3)-(+3) schemes show slightly improved squeezing relative to the SPS and (-1)$(+1)$ schemes. The final plot, Fig. 7-c), provides the change in phase noise, $\Delta \phi_{\text {out }}$ as it varies with $\Delta M a g_{\text {in }}$ and hence shows the extent of ANPNC each scheme exhibits. Reflecting the constellation plots, the $(-1)-(+1)$ scheme results in the largest
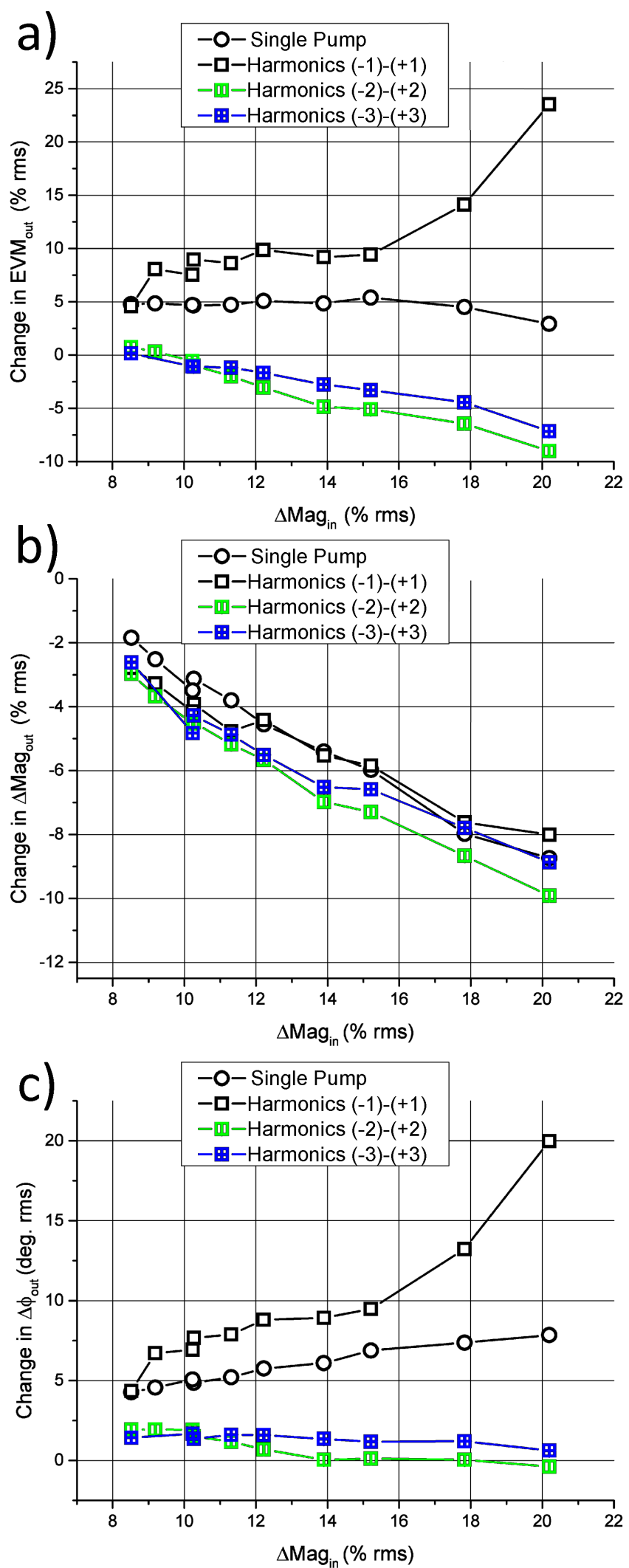

Fig. 7. Plots of the change in noise statistics relative to the noise loaded back to back for the Single Pump, $(-1)-(+1),(-2)-(+2)$ and $(-3)-(+3)$ cases: a) Change in EVM; b) Change in $\Delta M a g_{\text {in }}$; c) Change in $\Delta \Phi_{\text {out }}$. 

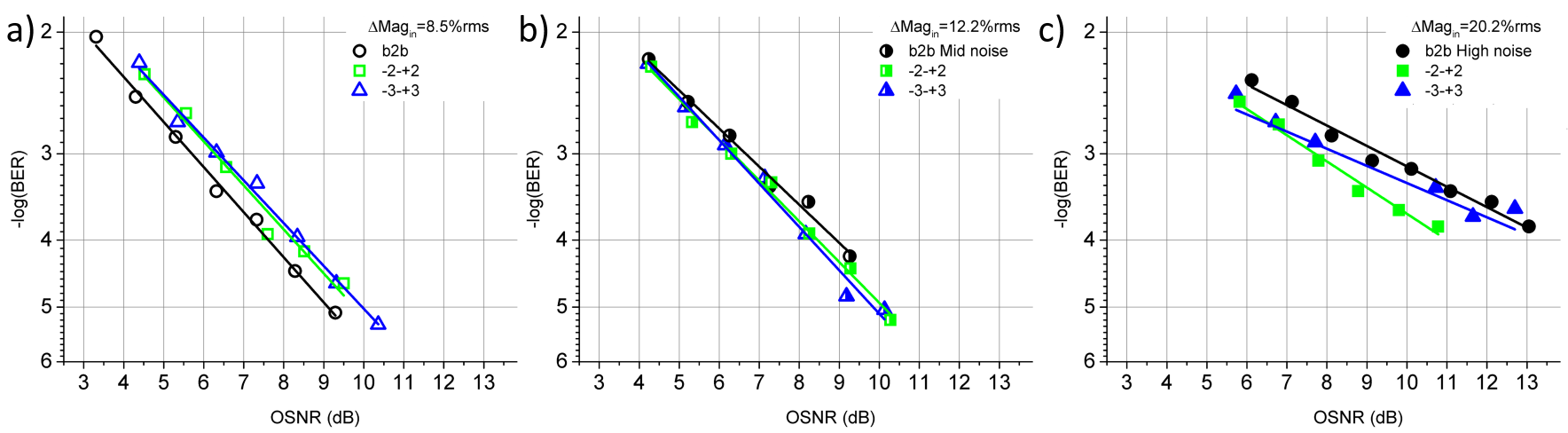

Fig. 8. Plots of BER as it varies with OSNR for the three considered noise cases: $8.5 \%$ rms. (open symbols), $12.2 \%$ rms. (half-filled symbols) and $20.2 \%$ rms. (filled symbols). Results are presented for the back to back case (circles), $(-2)-(+2)$ case (squares) and the $(-3)-(+3)$ case (triangles).

increase in $\Delta \phi_{\text {out }}$ which only becomes worse with increasing $\Delta M a g_{i n}$. This is followed by the SPS scheme which likewise shows a deterioration in $\Delta \Phi_{\text {out }}$ which becomes worse with $\Delta M_{a g}$ in but at a slower rate. Finally, the $(-2)-(+2)$ and ($3)-(+3)$ schemes show the smallest increase in $\Delta \Phi_{\text {out }}$. This degradation decreases with increasing $\Delta M a g_{i n}$ and indeed constitutes an improvement in $\Delta \Phi_{\text {out }}$ for the highest noise levels studied. This improvement is due to the chirp induced by the noise loading MZM being undone by the SPM in the scheme.

Lastly, in Fig. 8, we provide BER plots (without the use of FEC) to confirm the functioning of the scheme. The same three noise cases considered in the constellation plots in Fig. 6 and three scenarios are considered: back to back, ($2)-(+2)$ and $(-3)-(+3)$. BERs were not taken for the SPS and $(-1)-(+1)$ schemes as, given the results presented in Fig. 6 and Fig. 7, they would be predictably much worse than (-2)-(+2) and (-3)-(+3) cases. For the lowest noise case (Fig. 8-a), $\Delta M a g_{i n}=8.5 \%$ rms., the regenerated signals for both the (-2)-(+2) and (-3)-(+3) cases (open green squares and open blue triangles, respectively) can be seen to have $\approx 0.75 \mathrm{~dB}$ worse receiver sensitivity than the back to back case (open black circles). In the intermediate noise case, $\Delta \mathrm{Mag}_{\text {in }}=12.2 \% \mathrm{rms}$. (Fig. 8-b), the (-2)-(+2) and (-3)$(+3)$ systems (half-filled green squares and half-filled blue triangles, respectively) result in a $\approx 0.5 \mathrm{~dB}$ improvement in receiver sensitivity for a BER of $10^{-4}$. Finally, for the highest noise case (Fig. 8-c), $\Delta$ Mag $_{i n}=20.2 \%$ rms., the (-2)-(+2) case can be seen to offer an improvement in receiver sensitivity of $\approx 1.5 \mathrm{~dB}$ for a BER of $10^{-3}$, whilst the (-3)-(+3) case only offers a $\approx 1 \mathrm{~dB}$ improvement. This mirrors the $E V M$ plot provided in Fig. 7-a) and is possibly due to a reduced OSNR in the (-3)-(+3) case relative to the $(-2)-(+2)$ on account of the higher required total launch power for saturation and hence higher ASE emitted by the pre-HNLF EDFA.

\section{CONCLUSION}

We have proposed a new concept for all-optical signal processing that relies on inter-tonal power transfer FWM and applied it to realise a 'pumpless' FWM based amplitude regenerator. The scheme allows us to deliver phase preserving amplitude regeneration without any additional pumps using an HNLF in which the standard, single pump scheme fails to do so. The elimination of the requirement for additional high power laser sources and stimulated Brillouin scattering suppression in exchange for a MZM and a PF (both of which can offer multichannel operation) may represent a large cost saving. This scheme not only enables simplified optical processing schemes, but also allows for the exploration of synthetic launch conditions which would not naturally occur in simple pump based systems and as such, we believe that it will facilitate further research into advanced processing schemes.

\section{REFERENCES}

[1] F. Wen, C. P. Tsekrekos, Y. Geng, X. Zhou, B. Wu, K. Qiu, S. K. Turitsyn, and S. Sygletos, "All-optical multilevel amplitude regeneration in a single nonlinear optical loop mirror," Opt. Express, vol. 26, no. 10, pp. 12698-12706, May 2018. [Online]. Available: http://www.opticsexpress.org/abstract.cfm?URI=oe-26-10-12698

[2] Z. Lali-Dastjerdi, M. Galili, H. C. H. Mulvad, H. Hu, L. K. Oxenløwe, K. Rottwitt, and C. Peucheret, "Parametric amplification and phase preserving amplitude regeneration of a $640 \mathrm{gbit} / \mathrm{s}$ rz-dpsk signal," Opt. Express, vol. 21, no. 22, pp. 25 944-25 953, Nov 2013. [Online]. Available: http://www.opticsexpress.org/abstract.cfm?URI=oe21-22-25944

[3] K. R. H. Bottrill, F. Parmigiani, D. J. Richardson, and P. Petropoulos, "Optical predistortion enabling phase preservation in optical signal processing demonstrated in fwm-based amplitude limiter," Journal of Lightwave Technology, vol. In Press, no. 99, pp. 1-1, 2016.

[4] A. Almaiman, Y. Cao, A. Mohajerin-Ariaei, M. Ziyadi, P. Liao, C. Bao, F. Alishahi, A. Fallahpour, B. Shamee, Y. Akasaka, T. Ikeuchi, S. Wilkinson, J. D. Touch, M. Tur, and A. E. Willner, "Phase-sensitive qpsk channel phase quantization by amplifying the fourth-harmonic idler using counter-propagating brillouin amplification," Optics Communications, vol. 423, pp. 48 - 52, 2018. [Online]. Available: http://www.sciencedirect.com/science/article/pii/S0030401818302736

[5] R. Slavík, F. Parmigiani, J. Kakande, C. Lundström, M. Sjdin, P. A. Andrekson, R. Weerasuriya, S. Sygletos, A. D. Ellis, L. Grüner-Nielsen, D. Jakobsen, S. Herstrøm, R. Phelan, J. O'Gorman, A. Bogris, D. Syvridis, S. Dasgupta, P. Petropoulos, and D. J. Richardson, "All-optical phase and amplitude regenerator for next-generation telecommunications systems," Nature Photonics, vol. 4, no. 10, pp. 690-695, Oct. 2010. [Online]. Available: http://www.nature.com/nphoton/journal/v4/n10/full/nphoton.2010.203.html

[6] S. Rahbarfam and S. Kumar, "Nonlinear phase noise reduction using digital back propagation and midpoint optical phase conjugation," Opt. Express, vol. 27, no. 6, pp. 8968-8982, Mar 2019. [Online]. Available: http://www.opticsexpress.org/abstract.cfm?URI=oe-27-6-8968

[7] F. Da Ros, A. Gajda, E. P. da Silva, A. Peczek, A. Mai, K. Petermann, L. Zimmermann, L. K. Oxenlwe, and M. Galili, "Optical phase conjugation in a silicon waveguide with lateral p-i-n diode for nonlinearity 
compensation," Journal of Lightwave Technology, vol. 37, no. 2, pp. 323-329, Jan 2019.

[8] J. Kakande, R. Slavík, F. Parmigiani, A. Bogris, D. Syvridis, L. Grüner-Nielsen, R. Phelan, P. Petropoulos, and D. J. Richardson, "Multilevel quantization of optical phase in a novel coherent parametric mixer architecture," Nature Photonics, vol. 5, no. 12, pp. 748-752, Dec. 2011. [Online]. Available: http://www.nature.com/nphoton/journal/v5/n12/full/nphoton.2011.254.htm

[9] G.-W. Lu, T. Sakamoto, and T. Kawanishi, "Wavelength conversion of optical 64qam through fwm in hnlf and its performance optimization by constellation monitoring," Opt. Express, vol. 22, no. 1, pp. 15-22, Jan 2014. [Online]. Available: http://www.opticsexpress.org/abstract.cfm?URI=oe-22-1-15

[10] H. N. Tan, T. Inoue, K. Solis-Trapala, S. Petit, Y. Oikawa, K. Ota, S. Takasaka, T. Yagi, M. Pelusi, and S. Namiki, "On the cascadability of all-optical wavelength converter for high-order qam formats," Journal of Lightwave Technology, vol. 34, no. 13, pp. 3194-3205, July 2016.

[11] A. E. Willner, A. Fallahpour, F. Alishahi, Y. Cao, A. Mohajerin-Ariaei, A. Almaiman, P. Liao, K. Zou, A. N. Willner, and M. Tur, "Alloptical signal processing techniques for flexible networks," Journal of Lightwave Technology, vol. 37, no. 1, pp. 21-35, Jan 2019.

[12] P. Minzioni, C. Lacava, T. Tanabe, J. Dong, X. Hu, G. Csaba, W. Porod, G. Singh, A. E. Willner, A. Almaiman, V. Torres-Company, J. Schrder, A. C. Peacock, M. J. Strain, F. Parmigiani, G. Contestabile, D. Marpaung, Z. Liu, J. E. Bowers, L. Chang, S. Fabbri, M. R. Vázquez, V. Bharadwaj, S. M. Eaton, P. Lodahl, X. Zhang, B. J. Eggleton, W. J. Munro, K. Nemoto, O. Morin, J. Laurat, and J. Nunn, "Roadmap on all-optical processing," Journal of Optics, vol. 21, no. 6, p. 063001, may 2019. [Online]. Available: https://doi.org/10.1088

[13] L. Yan, A. Willner, X. Wu, A. Yi, A. Bogoni, Z. Y. Chen, and H. Y. Jiang, "All-optical signal processing for UltraHigh speed optical systems and networks," Journal of Lightwave Technology, vol. 30, no. 24, pp. 3760-3770, Dec. 2012.

[14] M. E. Marhic, Fiber Optical Parametric Amplifiers, Oscillators and Related Devices. Cambridge University Press, 2007. [Online]. Available: http://dx.doi.org/10.1017/CBO9780511600265

[15] E. Lichtman, A. A. Friesem, R. G. Waarts, and H. H. Yaffe, "Exact solution of four-wave mixing of copropagating light beams in a kerr medium," J. Opt. Soc. Am. B, vol. 4, no. 11, pp. 1801-1805, Nov 1987. [Online]. Available: http://josab.osa.org/abstract.cfm?URI=josab4-11-1801

[16] K. R. H. Bottrill, G. Hesketh, F. Parmigiani, D. J. Richardson, and P. Petropoulos, "Optimisation of amplitude limiters for phase preservation based on the exact solution to degenerate four-wave mixing," Opt. Express, vol. 24, no. 3, pp. 2774-2787, Feb 2016 [Online]. Available: http://www.opticsexpress.org/abstract.cfm?URI=oe24-3-2774

[17] G. Hesketh, K. R. H. Bottrill, F. Parmigiani, D. J. Richardson, and P. Petropoulos, "On the role of signal-pump ratio in fwm-based phase preserving amplitude regeneration," in International Conference on Optical Networks ICTON '15, July 2015.

[18] K. R. H. Bottrill, N. Taengnoi, Y. Hong, D. J. Richardson, and P. Petropoulos, "Self-pumping saturated four wave mixing through harmonic synthesis," in Optical Communication (ECOC), 2019 European Conference on, Sept 2019, p. Tue.1.C.6.

[19] J. B. Coles, B. P.-P. Kuo, N. Alic, S. Moro, C.-S. Bres, J. M. C. Boggio, P. Andrekson, M. Karlsson, and S. Radic, "Bandwidthefficient phase modulation techniques for stimulated brillouin scattering suppression in fiber optic parametric amplifiers," Opt. Express, vol. 18, no. 17, pp. 18 138-18150, Aug 2010. [Online]. Available: http://www.opticsexpress.org/abstract.cfm?URI=oe-18-17-18138

[20] T. Inoue, K. Tanizawa, and S. Namiki, "Guard-band-less and polarization-insensitive tunable wavelength converter for phasemodulated signals: Demonstration and signal quality analyses," Journal of Lightwave Technology, vol. 32, no. 10, pp. 1981-1990, May 2014.

[21] A. Striegler, M. Meissner, K. Cvecek, K. Sponsel, G. Leuchs, and B. Schmauss, "Nolm-based rz-dpsk signal regeneration," IEEE Photonics Technology Letters, vol. 17, no. 3, pp. 639-641, March 2005.

[22] M. Sorokina, "Design of multilevel amplitude regenerative system," Opt. Lett., vol. 39, no. 8, pp. 2499-2502, Apr 2014. [Online]. Available: http://ol.osa.org/abstract.cfm?URI=ol-39-8-2499

[23] X. Huang, Z. Zhang, C. Qin, Y. Yu, and X. Zhang, "Optimized quantum well semiconductor optical amplifier for rz-dpsk signal regeneration," IEEE Journal of Quantum Electronics, vol. 47, no. 6, pp. 819-826, June 2011.

[24] L. Li, P. G. Patki, Y. B. Kwon, V. Stelmakh, B. D. Campbell, M. Annamalai, T. I. Lakoba, and M. Vasilyev, "All-optical regenerator of multi-channel signals," Nature Communications, vol. 8, no. 1, p. 884 , 2017. [Online]. Available: https://doi.org/10.1038/s41467-017-00874-0

[25] S. P. Singh, R. Gangwar, and N. Singh, "Nonlinear scattering effects in optical fibers," Progress in Electromagnetics Research, vol. 74, pp. 379-405, 2007.

[26] G. P. Agrawal, Nonlinear fiber optics. Amsterdam: Elsevier/Acad. Press, 2007.

[27] T. Kato, Y. Suetsugu, M. Takagi, E. Sasaoka, and M. Nishimura, "Measurement of the nonlinear refractive index in optical fiber by the cross-phase-modulation method with depolarized pump light," Opt. Lett., vol. 20, no. 9, pp. 988-990, May 1995. [Online]. Available: http://ol.osa.org/abstract.cfm?URI=ol-20-9-988

[28] M. Matsumoto, "Phase noise generation in an amplitude limiter using saturation of a fiber-optic parametric amplifier," Opt. Lett., vol. 33, no. 15, pp. 1638-1640, Aug 2008. [Online]. Available: http://ol.osa.org/abstract.cfm?URI=ol-33-15-1638 